\title{
Patient selection before endobronchial valve treatment
}

\author{
To the Editor:
}

We read with great interest the study by HERTH et al. [1]. They investigated the ability of the bronchoscopic Chartis Pulmonary Assessment System to predict the response to unidirectional endobronchial valve (EBV) treatment by determining the presence of collateral ventilation $(\mathrm{CV})$ and the Chartis system showed an accuracy level of $75 \%$ in predicting whether or not the target lobe volume reduction (TLVR) cut-off $(\geqslant 350 \mathrm{~mL})$ would be reached.

As previously reported [2], EBV treatment brought emphysema patients significant but modest improvement of forced expiratory volume in $1 \mathrm{~s}$ (FEV1), exercise capacity and quality of life compared with controls, while another type of unidirectional valve, intrabronchial valve treatment, did not [3]. However, only a subgroup of patients could benefit more from EBV treatment. Therefore, defining and selecting this subgroup of patients is of great help to improve the clinically significant benefit of EBV treatment. To date, three methods can be used before EBV placement to predict therapeutic effect, which are CV detected by Chartis [1], and heterogeneity and fissure completeness assessed by high-resolution computed tomography (HRCT) [2, 4, 5]. This raises two questions.

First, which of these three methods has the highest capability to predict response to EBV treatment? Regrettably, HeRTH et al. [1] did not provide information about heterogeneity and fissure completeness. GOMPELMANN et al. [6] studied whether the accuracy of CV assessment was comparable to fissure analysis from HRCT in predicting clinically significant lung volume reduction following EBV treatment. Preliminary results of this study showed that Chartis and HRCT matched 24 (77.4\%) times in 31 patients, and did not match seven $(22.6 \%)$ times. Complete results of this study were not available when we were reviewing the literature, so we could not know the difference in the accuracy of predicting response to EBV treatment between these two methods. Therefore, we tried to extract comparable data from other studies. HERTH et al. [1] reported that CV-negative patients had a median TLVR of $752.7 \mathrm{~mL}$ compared to $98.6 \mathrm{~mL}$ in CVpositive patients; $43 \%$ of $C V$-negative patients showed change in $(\Delta) \mathrm{FEV} 1 \geqslant 15 \%$ at 1 month from baseline after EBV treatment. SCIURBA et al. [2] reported patients with complete fissure had a median TLVR of $713 \mathrm{~mL}$ compared to $196 \mathrm{~mL}$ in patients with incomplete fissure; $42.6 \%$ of patients with complete fissure showed $\triangle \mathrm{FEV} 1 \geqslant 15 \%$ at 6 months from baseline after EBV treatment. HeRTH et al. [4] reported that $35.2 \%$ of patients with more heterogeneous emphysema showed $\Delta \mathrm{FEV} 1 \geqslant 15 \%$ at 6 months from baseline after EBV treatment. So, it seems that CV detected by Chartis and fissure completeness assessed by HRCT are comparable in predicting response to EBV treatment.

Second, does combining those two or three methods have a higher capability to predict response to EBV treatment than a single one? Data from Herth et al. [1] showed that the false-positive value and the false negative value for Chartis system were $38 \%$ and $12 \%$, respectively. LINDQUIST et al. [7] reported that nine out of 49 patients screened with HRCT had intact fissures and went onto Chartis assessment and EBV placement, seven of whom were CV-negative and two were CV-positive on Chartis. The CV-negative group showed clinically significant improvement of lung function and St George's Respiratory Questionnaire, while the CV-positive group did not. Thus, combining these methods may further improve the predictive value.

As discussed here, further studies are warranted to investigate these two questions, which may help build an effective workflow for patients selection before EBV treatment.

@ERSpublications

Combining Chartis and HRCT may help build an effective workflow for patients selection for endobronchial valve treatment http://ow.ly/qn0sn

Zhen Yang, Jie Chen and Liang An Chen

Dept of Respiratory Disease, Chinese PLA General Hospital, Beijing, China.

Correspondence: L.A. Chen, Dept of Respiratory Disease, Chinese PLA General Hospital, 28 Fuxing Road, Beijing, 100853, China. E-mail: chenla301@263.net 


\title{
References
}

1 Herth FJ, Eberhardt R, Gompelmann D, et al. Radiological and clinical outcomes of using Chartis ${ }^{\mathrm{TM}}$ to plan endobronchial valve treatment. Eur Respir J 2013; 41: 302-308.

2 Sciurba FC, Ernst A, Herth FJ, et al. A randomized study of endobronchial valves for advanced emphysema. $N$ Engl J Med 2010; 363: 1233-1244.

3 Ninane V, Geltner C, Bezzi M, et al. Multicentre European study for the treatment of advanced emphysema with bronchial valves. Eur Respir J 2012; 39: 1319-1925.

4 Herth FJ, Noppen M, Valipour A, et al. Efficacy predictors of lung volume reduction with Zephyr valves in a European cohort. Eur Respir J 2012; 39: 1334-1342.

5 Valipour A, Herth FJF, Burghuber OC, et al. Target lobe volume reduction and COPD outcome measures after endobronchial valve therapy. Eur Respir J 2014; 43: 387-396.

6 Gompelmann D, Eberhardt R, Slebos D-J, et al. Comparison between Chartis pulmonary assessment system detection of collateral ventilation $v s$ corelab CT fissure analysis for predicting atelectasis in emphysema patients treated with endobronchial valves. Eur Respir J 2011; 38: Suppl. 55, 631s-632s.

7 Lindquist S, Chung S, Peters M, et al. Chartis evaluation of collateral ventilation versus HRCT assessment, in predicting clinical outcomes following endobronchial valve therapy (EBV) in COPD patients. Eur Respir J 2012; 40: Suppl. 56, 864s.

\section{Infant lung function and wheeze in later childhood in the Southampton Women's Survey}

\author{
To the Editor:
}

We were interested to read the study by VAN DER GUGTEN et al. [1] reporting associations between increased neonatal respiratory resistance and wheezing illnesses during infancy, and between reduced neonatal respiratory compliance and wheezing illnesses during the first 5 years of life and late-onset and persistent wheeze phenotypes. Reduced respiratory compliance was also associated with asthma, defined both according to primary care consultations and prescriptions or referral for wheezing illnesses, and according to patient-reported symptoms and lung function at the age of 5 years. The authors proposed that compliance and resistance might reflect different lung characteristics that are associated with symptoms in different age periods. Our data from normal-term infants in the Southampton Women's Survey birth cohort provide further evidence that impaired physiological measurements soon after birth are associated with specific wheeze phenotypes. We previously reported an association between lower maximal flow at functional residual capacity $\left(V^{\prime} \max _{\mathrm{FRC}}\right)$ in early infancy and later transient wheeze [2]. The paper by VAN DER GUGTEN et al. [1] has led us to analyse our data further with regards to compliance of the respiratory system (Crs) and we have found that lower $C$ rs is associated with asthma in our cohort too. Using the raised volume thoracoabdominal compression technique, we also measured forced expiratory volume in $0.4 \mathrm{~s}$ (FEV0.4); lower FEV0.4 measurements were associated with increased childhood asthma risk.

We have previously described our methods [3]. In brief, lung function was measured between 5 and 14 weeks of age in 147 term infants. Infants were tested lying supine in quiet sleep, augmented by oral chloral hydrate $\left(75-100 \mathrm{mg} \cdot \mathrm{kg}^{-1}\right) . V^{\prime} \max _{\mathrm{FRC}}$ and $\mathrm{FEV} 0.4$ were calculated from partial and raised volume expiratory flow-volume curves, respectively. Crs was calculated from passive flow-volume curves following single occlusions. Wheeze data were collected at 6, 12, 24 and 36 months and 6 years using questions from the ISAAC (International Study of Asthma and Allergies in Childhood) core questionnaire wheezing module. 95 children provided questionnaire data and spirometry at age 6 years. Associations between infant lung function measurements and wheeze phenotype were assessed using regression; significant confounders were included in multivariable models (as described in [2]). Table 1 shows an association between lower early infancy $C r$ and childhood asthma together with an association between lower $V^{\prime} \max _{\mathrm{FRC}}$ and transient wheeze. The former confirms the finding of VAN DER GUGTEN et al. [1] of reduced early-life Crs in children later diagnosed with asthma and the latter; although not identical to the findings of VAN DER GUGTEN et al. [1], it is, however, consistent with their proposal that low airway calibre is a likely contributor to wheeze 